\title{
Testing non-linear force-free coronal magnetic field extrapolations with the Titov-Démoulin equilibrium (Research Note)
}

\author{
T. Wiegelmann ${ }^{1}$, B. Inhester ${ }^{1}$, B. Kliem ${ }^{2}$, G. Valori ${ }^{2}$, and T. Neukirch ${ }^{3}$ \\ 1 Max-Planck-Institut für Sonnensystemforschung, Max-Planck-Strasse 2, 37191 Katlenburg-Lindau, Germany \\ e-mail: wiegelmann@mps.mpg.de \\ 2 Astrophysical Institute Potsdam, An der Sternwarte 16, 14482 Potsdam, Germany \\ 3 School of Mathematics and Statistics, University of St. Andrews, St. Andrews, KY16 9SS, UK
}

Received 22 December 2005 / Accepted 14 March 2006

ABSTRACT

\begin{abstract}
Context. As the coronal magnetic field can usually not be measured directly, it has to be extrapolated from photospheric measurements into the corona.

Aims. We test the quality of a non-linear force-free coronal magnetic field extrapolation code with the help of a known analytical solution.

Methods. The non-linear force-free equations are numerically solved with the help of an optimization principle. The method minimizes an integral over the force-free and solenoidal condition. As boundary condition we use either the magnetic field components on all six sides of the computational box in Case I or only on the bottom boundary in Case II. We check the quality of the reconstruction by computing how well force-freeness and divergence-freeness are fulfilled and by comparing the numerical solution with the analytical solution. The comparison is done with magnetic field line plots and several quantitative measures, like the vector correlation, Cauchy Schwarz, normalized vector error, mean vector error and magnetic energy.

Results. For Case I the reconstructed magnetic field shows good agreement with the original magnetic field topology, whereas in Case II there are considerable deviations from the exact solution. This is corroborated by the quantitative measures, which are significantly better for Case I.

Conclusions. Despite the strong nonlinearity of the considered force-free equilibrium, the optimization method of extrapolation is able to reconstruct it; however, the quality of reconstruction depends significantly on the consistency of the input data, which is given only if the known solution is provided also at the lateral and top boundaries, and on the presence or absence of flux concentrations near the boundaries of the magnetogram.
\end{abstract}

Key words. Sun: magnetic fields - Sun: corona - Sun: photosphere

\section{Introduction}

Several methods have been proposed to compute the non-linear force-free coronal magnetic field in active regions from measurements of the photospheric magnetic field vector, e.g. the direct upward integration method (Wu et al. 1990), the GradRubin method (e.g. Sakurai 1981; Amari et al. 1999; Régnier et al. 2002; Bleybel et al. 2002; Wheatland 2004; Amari et al. 2006; Inhester \& Wiegelmann 2006), the Green's function like methods (Yan \& Sakurai 2000), the stress and relax method (Roumeliotis 1996; Valori et al. 2005) and the optimization method (Wheatland et al. 2000; Wiegelmann 2004; Wiegelmann et al. 2006).

A standard test for force-free extrapolation methods is the application of the codes to known analytical or numerical nonlinear force-free equilibria. Due to the general mathematical difficulty of the problem, only very few such solutions are known. The comparison of numerical nonlinear force-free extrapolation codes with the class of nonlinear force-free equilibria found by Low \& Lou (1990, LL from here on) has in the past ten years emerged as a certain standard test. The LL equilibria are a class of axisymmetric equilibria which are separable in spherical coordinates. They are self-similar in the radial coordinate, and the polar angle dependence is determined from a non-linear eigenvalue equation. The symmetry is broken by cutting out a rectangular chunk of the solution by using a Cartesian coordinate system which is shifted and rotated with respect to the original coordinate system in which the LL equilibria are calculated. The parameters of the LL solutions and the parameters of the new Cartesian coordinate system allow for a large number of different situations which can be used for tests.

Nevertheless, it is highly desirable to also use tests different from LL. A first step has been made by Valori et al. (2005), who used an equilibrium taken from a numerical investigation of a twisted flux rope. Valori \& Kliem (2005, private communication) have further tested their nonlinear force-free extrapolation code with configurations containing a flux rope by considering the approximate analytical force-free equilibrium established by (Titov \& Démoulin 1999, henceforth TD). In a recent review talk on nonlinear force-free extrapolation, T. Neukirch also strongly recommended the use of other equilibria, in particular the TD-equilibrium, to test the quality of non-linear force-free extrapolation codes. The TD equilibrium has a much more concentrated current density than LL, and the magnetogram is more structured. Also, the final equilibrium has a different topology than the corresponding potential field, which is not true for LL. 
Table 1. Parameter set of the TD equilibrium.

\begin{tabular}{|c|c|c|}
\hline $\bar{R}$ & $=110 \mathrm{Mm}=2.2$ & (major torus radius) \\
\hline$a$ & $=35 \mathrm{Mm}=0.7$ & (minor torus radius) \\
\hline$d$ & $=50 \mathrm{Mm}=1.0$ & (depth of torus center) \\
\hline$L$ & $=100 \mathrm{Mm}=2.0$ & (monopole distance) \\
\hline$q$ & $=100 \mathrm{~T} \mathrm{Mm}^{2}$ & (magnetic charge) \\
\hline$I_{0}$ & $=-13 \mathrm{TA}$ & (line current) \\
\hline$I$ & $=3.391 \mathrm{TA}$ & (ring current) \\
\hline$h_{\text {apex }}$ & $=1.2$ & (apex height) \\
\hline$y_{\mathrm{foo}}$ & $=1.960$ & (footpoint position) \\
\hline$B_{\text {ap }}$ & $=1.0$ & (norm. apex field strength) \\
\hline$\langle\Phi\rangle$ & $=-1.41 \pi$ & (average twist) \\
\hline
\end{tabular}

The aim of this research note is to study the performance the optimization method in reconstructing the TD equilibrium. The optimization code has so far only been tested with the LL solution with rather small resolution $(40 \times 40 \times 20)$ in Wheatland et al. (2000), on $80 \times 80 \times 40$ grids in Wiegelmann $\&$ Neukirch (2003) and on a $64^{3}$ grid as part of a comparison of six different extrapolation methods in Schrijver et al. (2006). The optimization code in the implementation of Wiegelmann (2004) was the fastest-converging and best-performing model of the six tested extrapolation codes. Here we test this optimization code with the TD equilibrium using a high numerical resolution $(150 \times 250 \times 100)$.

\section{The TD equilibrium}

Like the LL solutions, the TD equilibrium is an axisymmetric equilibrium and the symmetry is broken by choosing the boundaries appropriately. The TD equilibrium has originally been constructed as a model of an active region containing a current- carrying flux tube. The model has later been used in a number of investigations of the initiation of CME eruptions (e.g., Roussev et al. 2003; Török et al. 2004; Kliem et al. 2004; Török \& Kliem 2005; Williams et al. 2005).

The axis of symmetry of the TD equilibrium is placed at a distance $d$ beneath the lower boundary (the photosphere). A line current of strength $I_{0}$ runs along the line of symmetry, which creates a potential magnetic field with circular field lines. Titov \& Démoulin (1999) then added a toroidal nonlinear force-free current around the axis of symmetry with minor radius $a$ and major radius $R$ and total current $I$, assuming $a \ll R$ (see also Fig. 2 in Titov \& Démoulin 1999). Similar to a tokomak, the ring current would not be in equilibrium with the potential field created by the line current. Therefore, two magnetic monopoles (strength $q$ ) of opposite polarity are placed on the symmetry axis at a distance $L$ from each other, with the force-free ring current half-way between them. The monopoles create a potential poloidal magnetic field which has field lines lying above the force-free ring current and thus holding it down. In this way a stable equilibrium situation can be achieved for certain parameter combinations (unstable cases have been investigated in the studies of CME initiation mentioned above).

For the present investigation we use the TD equilibrium (given by their Eqs. (16)-(22) and (31)) with the set of parameters given in Table 1. This yields a stable equilibrium because the twist of the flux rope is clearly sub-critical with respect to the kink instability (Török et al. 2004) and the monopoles are placed sufficiently distant (Titov \& Démoulin 1999). In Table 1 we have also included the derived parameters of the apex height of the ring current above the photosphere $(=R-d)$, the footpoint position of the ring current $\left(=\sqrt{R^{2}-d^{2}}\right)$, the average field line twist in the nonlinear force-free ring current (averaged over the toroidal cross section), and the normalization of the field and of the various length parameters.

The TD equilibrium has a number of properties which we mention here explicitly because they are relevant for magnetic field extrapolation methods. The artificial magnetogram of the TD equilibrium basically has a large-scale bipolar structure (due to the line current), but this structure is modified by the monopoles and the ring current to an almost quadrupolar structure. Because the line current extends from $-\infty$ to $\infty$, the magnetic field on the photosphere does not drop to zero with larger distance from the nonlinear force-free region in the direction of the line current. This is different from the LL solutions, for which the photospheric magnetic field can be made very small at the edges of the magnetogram if the computational box is chosen large enough. On the other hand, the coronal current density (and therefore the region with non-vanishing $\alpha$ on the photospheric boundary) is strongly concentrated for the TD equilibrium (to the region of the ring current). In the LL case $\alpha$ is distributed over extended regions of the lower boundary. Finally, as already mentioned above, the TD equilibrium magnetic field has a different topology than the corresponding potential magnetic field calculated from the $B_{z}$ component of the magnetic field on the lower boundary. This is not the case for the standard LL cases usually used for testing nonlinear force-free extrapolation codes.

\section{Method}

Force-free coronal magnetic fields have to obey the equations

$$
\begin{aligned}
(\nabla \times \boldsymbol{B}) \times \boldsymbol{B} & =\mathbf{0}, \\
\nabla \cdot \boldsymbol{B} & =0 .
\end{aligned}
$$

We solve these equations with the help of an optimization principle, as proposed by Wheatland et al. (2000) and generalized by Wiegelmann \& Inhester (2003). We define the functional

$$
L=\int_{V} w(x, y, z)\left[B^{-2}|(\nabla \times \boldsymbol{B}) \times \mathbf{B}|^{2}+|\nabla \cdot \boldsymbol{B}|^{2}\right] \mathrm{d}^{3} x
$$

where $w(x, y, z)$ is a weighting function. It is obvious that (for $w>0)$ the force-free Eqs. (1)-(2) are fulfilled when $L$ equals zero. We compute the magnetic field in a box with $n x=150$, $n y=250$ and $n z=100$ points. The numerical method works as follows. As an initial configuration we compute a potential magnetic field in the whole box with the help of a Green's function method as described in Aly (1989). The next step is slightly different for the two cases.

- Case I: we impose the exact TD magnetic field vector on all six boundaries of the computational box. The weighting function is $w=1$ in the entire box.

- Case II: we impose the exact TD magnetic field vector only on the bottom boundary (photosphere). The boundary conditions on the lateral and top boundaries of the computational box are given by the initial potential field. The weighting function is $w=1$ in the center $118 \times 218 \times 84$ region and drops to 0 with a cosine profile in a 16-pixel boundary layer towards the lateral and top boundaries of the computational box.

In both cases we iterate for the magnetic field inside the computational box by minimizing Eq. (3). The program is written in $\mathrm{C}$ and has been parallelized with OpenMP. The computations have been done on 8 Procs. The details of the current implementation of our code are described in Wiegelmann (2004). 
Table 2. Figures of merit for the comparison of our reconstruction with the original TD equilibrium. See Sect. 4 for details. The diagnostic was done on the center $118 \times 218 \times 84$ grid and a boundary layer of 16 points towards the lateral and top boundaries of the computational box has been omitted.

\begin{tabular}{|c|c|c|c|c|c|c|c|c|c|c|c|c|c|}
\hline Model & $L_{\text {inner }}$ & $L_{1 \text { inner }}$ & $L_{2 \text { inner }}$ & $\|\nabla \cdot \boldsymbol{B}\|_{\infty}$ & $\|\boldsymbol{j} \times \boldsymbol{B}\|_{\infty}$ & $C_{\mathrm{vec}}$ & $C_{\mathrm{CS}}$ & $E_{N}^{\prime}$ & $E_{M}^{\prime}$ & $\epsilon$ & $\epsilon_{\mathrm{P}}$ & It. Steps & comp. Time \\
\hline T\&D & 0.015 & 0.013 & 0.002 & 3.13 & 5.54 & 1 & 1 & 1 & 1 & 1 & 2.36 & - & - \\
\hline Potential & 0.26 & $5.5 \times 10^{-8}$ & 0.26 & 11.89 & 0.076 & 0.82 & 0.82 & 0.39 & 0.35 & 0.42 & 1 & - & $22 \min$ \\
\hline Case I & 0.0016 & 0.0012 & 0.0004 & 1.92 & 2.36 & 0.9996 & 0.9998 & 0.99 & 0.99 & 0.999 & 2.36 & 22180 & $6 \mathrm{~h} 37 \mathrm{~min}$ \\
\hline Case II & 0.37 & 0.34 & 0.02 & 2.88 & 10.48 & 0.96 & 0.94 & 0.66 & 0.58 & 0.66 & 1.56 & 5330 & $1 \mathrm{~h} 35 \mathrm{~min}$ \\
\hline
\end{tabular}
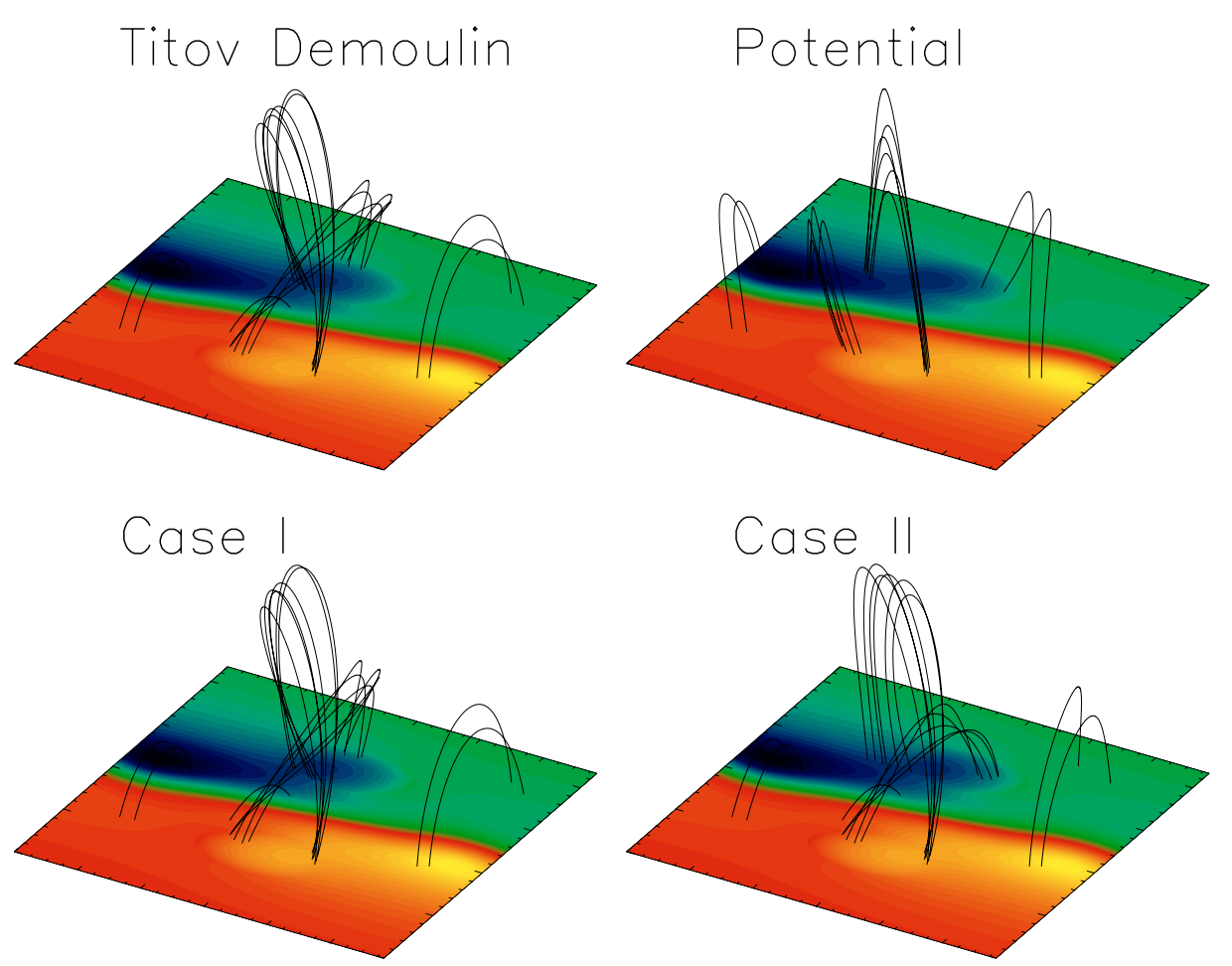

Fig. 1. We compare the magnetic field structure of the original Titov Demoulin equilibrium (Titov Demoulin) with a potential field extrapolation (Potential), a non-linear force-free extrapolation with all six boundary surfaces prescribed (Case I) and a non-linear force-free extrapolation which uses only the photospheric magnetic field data (Case II). The panels show the center $118 \times 218 \times 84$ region of the original $150 \times 250 \times 100$. The colour coding shows the magnetic field strength on the photosphere. We used the same start points for the magnetic field line computation in the positive (yellow) magnetic field region on the photosphere in all panels.

\section{Figures of merit}

To assess the quality of our reconstruction we introduce several figures of merit in Table 2. The first column of the table gives the name of model, column two contains the integral $L=L_{1}+L_{2}$ defined in Eq. (3), the third column $L_{1}$ shows how good the forcefree condition (first term of the integral in Eq. (3)) is fulfilled and the forth column $L_{2}$ corresponds to the solenoidal condition (second term of the integral in Eq. (3)). $L, L_{1}$ and $L_{2}$ are calculated with the magnetic field normalized by the average magnetic field strength on the photosphere and the length scale normalized by the average box length. We emphasize, however, that we restrict the integration domain to the inner part of the computational box, excluding the boundary layer in which $w(x, y, z) \neq 1$. Thus strictly speaking the diagnostic quantities differ from the integrals given in Eq. (3). We therefore call them $L_{\text {inner }}, L_{1}$ inner and $L_{2 \text { inner. }}$. Columns five to eleven contain quantitative measures on how good the reconstructed solution agrees with the original Titov \& Demoulin equilibrium. These figures of merit have been introduced by Schrijver et al. (2006) to compare the results of six different extrapolation codes (including the code used here) with the Low \& Lou solution. In a coordinated study Amari et al. (2006) used the same figures of merit to compare the reconstruction of two Grad-Rubin codes also with the Low $\&$ Lou solution. These figures quantify the agreement between vector fields $\boldsymbol{B}$ (Titov \& Demoulin equilibrium) and $\boldsymbol{b}$ (reconstructed fields). We use exactly the same definitions as given in
Sect. 4 of Schrijver et al. (2006), in order to allow also an comparison of how well our code reconstructs the Low \& Lou solution (see Tables I and II in Schrijver et al. 2006) and the Titov \& Demoulin solution (Table 2 here).

Column five contains the $L_{\infty}$ norm of the divergence of the magnetic field

$\|\nabla \cdot \boldsymbol{B}\|_{\infty}=\sup _{\boldsymbol{x} \in V}|\nabla \cdot \boldsymbol{B}|$.

Column six contains the $L_{\infty}$ norm of the Lorentz force of the magnetic field

$\|\boldsymbol{j} \times \boldsymbol{B}\|_{\infty}=\sup _{\boldsymbol{x} \in V}|\boldsymbol{j} \times \boldsymbol{B}|$.

Column seven contains the vector correlation

$C_{\mathrm{vec}}=\sum_{i} \boldsymbol{B}_{\boldsymbol{i}} \cdot \boldsymbol{b}_{\boldsymbol{i}} /\left(\sum_{i}\left|\boldsymbol{B}_{i}\right|^{2} \sum_{i}\left|\boldsymbol{b}_{\boldsymbol{i}}\right|^{2}\right)^{1 / 2}$,

Col. eight the Cauchy-Schwarz inequality

$C_{\mathrm{CS}}=\frac{1}{N} \sum_{i} \frac{\boldsymbol{B}_{\boldsymbol{i}} \cdot \boldsymbol{b}_{\boldsymbol{i}}}{\left|\boldsymbol{B}_{\boldsymbol{i}}\right|\left|\boldsymbol{b}_{\boldsymbol{i}}\right|}$,

where $N$ is the number of vectors in the field. The normalized vector error is defined as

$E_{\mathrm{N}}=\sum_{i}\left|\boldsymbol{b}_{\boldsymbol{i}}-\boldsymbol{B}_{i}\right| / \sum_{i}\left|\boldsymbol{B}_{i}\right|$ 

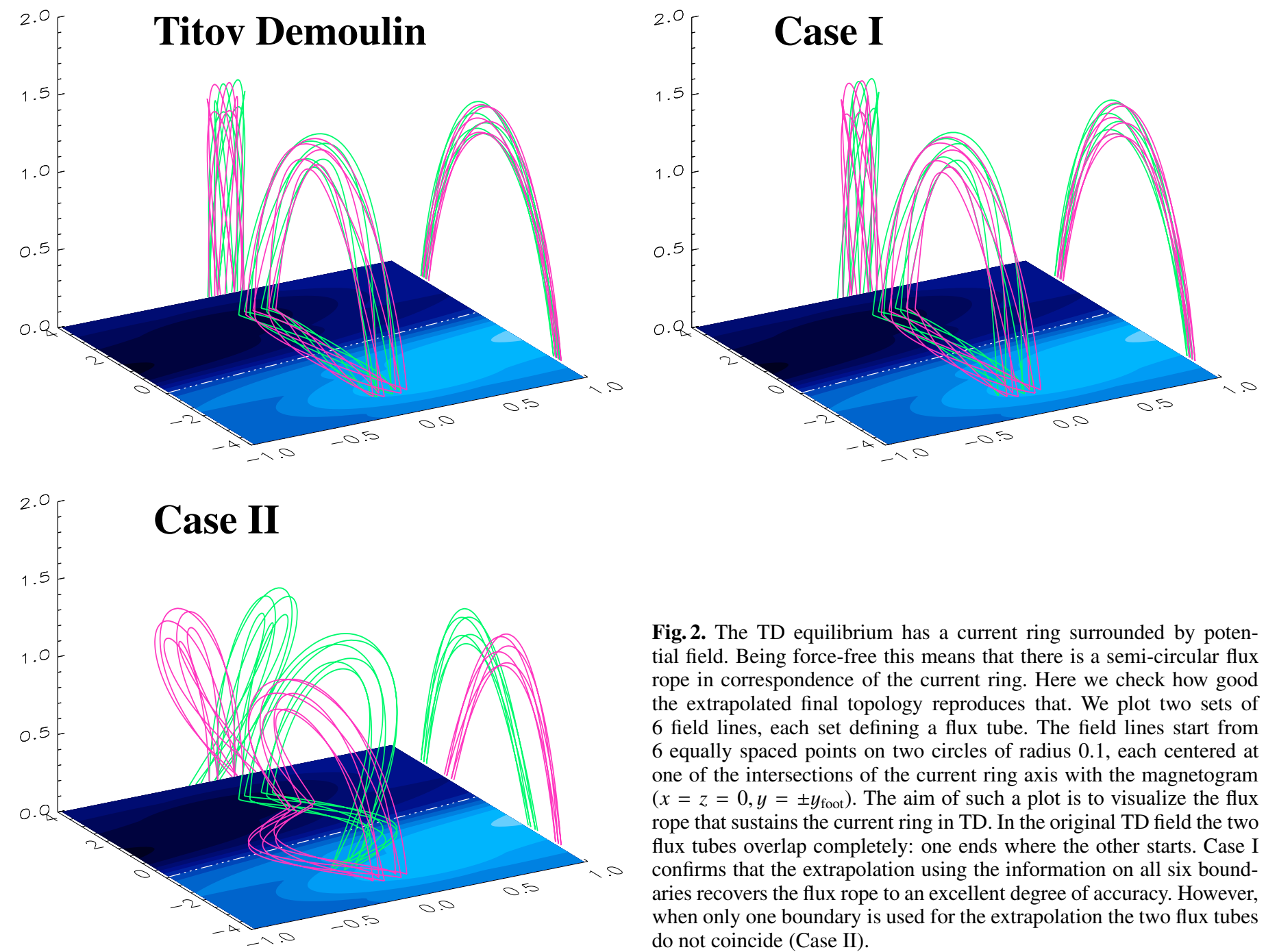

Fig. 2. The TD equilibrium has a current ring surrounded by potential field. Being force-free this means that there is a semi-circular flux rope in correspondence of the current ring. Here we check how good the extrapolated final topology reproduces that. We plot two sets of 6 field lines, each set defining a flux tube. The field lines start from 6 equally spaced points on two circles of radius 0.1 , each centered at one of the intersections of the current ring axis with the magnetogram $\left(x=z=0, y= \pm y_{\text {foot }}\right)$. The aim of such a plot is to visualize the flux rope that sustains the current ring in TD. In the original TD field the two flux tubes overlap completely: one ends where the other starts. Case I confirms that the extrapolation using the information on all six boundaries recovers the flux rope to an excellent degree of accuracy. However, when only one boundary is used for the extrapolation the two flux tubes do not coincide (Case II).

and the mean vector error as

$E_{\mathrm{M}}=\frac{1}{N} \frac{\sum_{i}\left|\boldsymbol{b}_{\boldsymbol{i}}-\boldsymbol{B}_{\boldsymbol{i}}\right|}{\sum_{i}\left|\boldsymbol{B}_{\boldsymbol{i}}\right|}$.

Columns nine and ten contain $E_{\mathrm{N}}^{\prime}=1-E_{\mathrm{N}}$ and $E_{\mathrm{M}}^{\prime}=1-$ $E_{\mathrm{M}}$, respectively. In column eleven we show the total magnetic energy of the reconstructed field normalized with the energy of the input field

$\epsilon=\frac{\sum_{i}\left|\boldsymbol{b}_{i}\right|^{2}}{\sum_{i}\left|\boldsymbol{B}_{i}\right|^{2}}$

The two vector fields agree perfectly if the figures of merit $\left(C_{\mathrm{vec}}, C_{\mathrm{CS}}, E_{\mathrm{N}}^{\prime}, E_{\mathrm{M}}^{\prime}, \epsilon\right)$ are unity. In the twelves column we list $\epsilon_{\mathrm{P}}$ which is the magnetic energy normalized by the energy of the corresponding potential field. Column thirteen contains the number of iteration steps until convergence and column fourteen the computing time on 8 processors.

\section{Results}

In Fig. 1 we compare the original TD equilibrium with a potential field extrapolation (Potential), a non-linear force-free extrapolation with all six boundaries prescribed (Case I) and a nonlinear force free extrapolation where only the bottom boundary

has been prescribed (Case II). The potential field does obviously not agree with the original. Case I shows a very good agreement with the original. Case II reconstructs the magnetic field approximately. Deviations of the magnetic field lines between Case II and the original are visible, in particular for field lines emerging in the nonlinear force-free region of the bottom boundary.

The visible inspection of Fig. 1 is supported by the figures of merit in Table 2. The figures of merit (Cols. 7-11) are for Case I better than $1 \%$ for all figures, mostly even better than $0.1 \%$. The integral force and divergence free conditions (Cols. 1-3) are even one order of magnitude better than the discretization error of the exact solution. The $L_{\infty}$ norms (Cols. 4 and 5) are of the same order of magnitude as the the discretization error.

The reason is that the optimization code minimizes $L$ with respect to the numerical grid. For Case II, on the contrary, $L$ is one order of magnitude higher than the discretization error of the exact solution. The reason is that for Case II an inconsistency exists between the bottom boundary and the other boundaries, where the initial potential field is kept. Correspondingly, the figures of merit are less good than in Case I.

The lateral and upper boundaries try to feed information about the original potential field into the solution, and because the potential field has a different topology, the flux rope of the 
TD equilibrium is not correctly reproduced in Case II. Figure 2 shows that the field lines calculated from start points lying on symmetric circles at the footpoints of the flux rope do outline a single flux rope only in Case I, but outline two flux ropes that separate with height in Case II.

\section{Conclusions}

We used the TD equilibrium to test a non-linear force-free optimization code. In Case I we prescribed all six boundaries of the computational box and in Case II only the bottom boundary. For Case I we get an almost perfect agreement with the original, and for Case II also a reasonable agreement. Case I is the real performance test of the code, because only if the correct boundary conditions on all boundaries are prescribed we have exactly the same physical problem and can expect to find the exact solution. For the reconstruction of magnetic fields from observed vector magnetograms the results of Case II are more important, however, because for real active regions the lateral boundaries are unknown. Let us also remark that the figures of merit here are comparable with the figures of merit in the LL case (Table 1 rows b) in Schrijver et al. (2006). For Cases II the results for TD are slightly worse for $L, L_{1}, C_{\mathrm{vec}}, E_{\mathrm{N}}^{\prime}$ and somewhat better for $L_{2}, C_{\mathrm{CS}}, E_{\mathrm{M}}^{\prime}$ compared with the LL case. The reconstructed magnetic energy in the TD case discussed here is not as accurate (only for Case II) as for the LL case. One has to consider, however, that the magnetic energy of the corresponding LL field (Case II in Schrijver et al. 2006) is only 1.10 times the potential field, where the TD equilibrium has a magnetic energy which is 2.36 higher than the potential field. The magnetic field strength on the photosphere for the LL field becomes very low close to the lateral boundaries, which is not the case for TD. Despite these difficulties, our code reconstructed the magnetic field topology approximately correct, even if the lateral boundaries are unknown. Current vectormagnetograms often have a limited field of view and consequently also significant magnetic flux close to the lateral boundaries. A comparison of direct measurements of chromospheric magnetic loops by Solanki et al. (2003) with a non-linear force-free extrapolation from a photospheric vectormagnetogram with a limited field of view in
Wiegelmann et al. (2005) showed a reasonable agreement, except for loops close to boundaries of the magnetogram.

Acknowledgements. The work of T. Wiegelmann was supported by DLR-grant 50 OC 0501 and a British Council-DAAD grant at the University of St. Andrews. T. Wiegelmann acknowledges the warm hospitality of the St. Andrews Solar Theory Group during two research visits. B. Kliem and G. Valori were supported by grants DFG MA 1376/16-2 and DFG HO 1424/9-1, respectively. We acknowledge useful discussions during two workshops on non-linear force-free field extrapolation methods organized by Karel Schrijver in Palo Alto 2004 and 2005. We thank the referee, Tahar Amari, for useful remarks to improve this paper.

\section{References}

Aly, J. J. 1989, Sol. Phys., 120, 19

Amari, T., Boulmezaoud, T. Z., \& Aly, J. J. 2006, A\&A, 446, 691

Amari, T., Boulmezaoud, T. Z., \& Mikic, Z. 1999, A\&A, 350, 1051

Bleybel, A., Amari, T., van Driel-Gesztelyi, L., \& Leka, K. D. 2002, A\&A, 395, 685

Inhester, B., \& Wiegelmann, T. 2006, Sol. Phys., 235, 201

Kliem, B., Titov, V. S., \& Török, T. 2004, A\&A, 413, L23

Low, B. C., \& Lou, Y. Q. 1990, ApJ, 352, 343

Régnier, S., Amari, T., \& Kersalé, E. 2002, A\&A, 392, 1119

Roumeliotis, G. 1996, ApJ, 473, 1095

Roussev, I. I., Forbes, T. G., Gombosi, T. I., et al. 2003, ApJ, 588, L45

Sakurai, T. 1981, Sol. Phys., 69, 343

Schrijver, C. J., DeRosa, M. L., Metcalf, T. R., et al. 2006, Sol. Phys., 235, 161 Solanki, S. K., Lagg, A., Woch, J., Krupp, N., \& Collados, M. 2003, Nature, 425, 692

Titov, V. S., \& Démoulin, P. 1999, A\&A, 351, 707

Török, T., \& Kliem, B. 2005, ApJ, 630, L97

Török, T., Kliem, B., \& Titov, V. S. 2004, A\&A, 413, L27

Valori, G., Kliem, B., \& Keppens, R. 2005, A\&A, 433, 335

Wheatland, M. S. 2004, Sol. Phys., 222, 247

Wheatland, M. S., Sturrock, P. A., \& Roumeliotis, G. 2000, ApJ, 540, 1150

Wiegelmann, T. 2004, Sol. Phys., 219, 87

Wiegelmann, T., \& Inhester, B. 2003, Sol. Phys., 214, 287

Wiegelmann, T., \& Neukirch, T. 2003, Nonlinear Process. Geophys., 10, 313

Wiegelmann, T., Inhester, B., \& Sakurai, T. 2006, Sol. Phys., 233, 215

Wiegelmann, T., Lagg, A., Solanki, S. K., Inhester, B., \& Woch, J. 2005, A\&A, 433, 701

Williams, D. R., Török, T., Démoulin, P., van Driel-Gesztelyi, L., \& Kliem, B. 2005, ApJ, 628, L163

Wu, S. T., Sun, M. T., Chang, H. M., Hagyard, M. J., \& Gary, G. A. 1990, ApJ, 362, 698

Yan, Y., \& Sakurai, T. 2000, Sol. Phys., 195, 89 